\title{
Intercalated Duct
}

National Cancer Institute

\section{Source}

National Cancer Institute. Intercalated Duct. NCI Thesaurus. Code C32820.

One of two types of intercalated ducts located in either the pancreas or salivary gland. 